\title{
The Crucial Role of C18-Cer in Fat-Induced Skeletal Muscle Insulin Resistance
}

\author{
Agnieszka U. Blachnio-Zabielska ${ }^{a}$ Marta Chacinska ${ }^{a}$ Mikkel H. Vendelbo ${ }^{b}$ \\ Piotr Zabielskic,d \\ aDepartment of Hygiene, Epidemiology and Metabolic Disorders, Medical University of Bialystok, \\ Bialystok, Poland; bepartment of Nuclear Medicine \& PET Center, Aarhus University Hospital, \\ Aarhus, Denmark; 'Department of Medical Biology, Medical University of Bialystok, Bialystok, Poland; \\ dDepartment of Physiology, Medical University of Bialystok, Bialystok, Poland
}

\section{Key Words}

Ceramide • Insulin resistance • Skeletal muscle

\begin{abstract}
Background/Aims: Muscle bioactive lipids accumulation leads to several disorder states. The most common are insulin resistance (IR) and type 2 diabetes. There is an ongoing debate which of the lipid species plays the major role in induction of muscle IR. Our aim was to elucidate the role of particular lipid group in induction of muscle IR. Methods: The analyses were performed on muscle from the following groups of rats: 1 . Control, fed standard diet, 2 HFD, fed high fat diet, 3. HFD/Myr, fed HFD and treated with myriocin (Myr), an inhibitor of ceramide de novo synthesis. We utilized $\left[\mathrm{U}^{13} \mathrm{C}\right]$ palmitate isotope tracer infusion and mass spectrometry to measure content and synthesis rate of muscle long-chain acyl-CoA (LCACoA), diacylglycerols (DAG) and ceramide (Cer). Results: HFD led to intramuscular accumulation of LCACoA, DAG and Cer and skeletal muscle IR. Myr-treatment caused decrease in Cer (most noticeable for stearoyl-Cer and oleoyl-Cer) and accumulation of DAG, possibly due to rechanneling of excess of intramuscular LCACoA towards DAG synthesis. An improvement in insulin sensitivity at both systemic and muscular level coincided with decrease in ceramide, despite elevated intramuscular DAG. Conclusion: The improved insulin sensitivity was associated with decreased muscle stearoyl- and oleoyl-ceramide content. The results indicate that accumulation of those ceramide species has the greatest impact on skeletal muscle insulin sensitivity in rats.

\section{Introduction}

High fat diet (HFD) consumption and obesity leads to accumulation of intramuscular lipids that are known to be associated with chronic and severe medical problems including insulin resistance (IR) and type 2 diabetes (T2D). Although it is clear that consumption of HFD leads to IR via intramuscular lipid accumulation, there is an ongoing debate which lipid 
class is the most important in triggering skeletal muscle insulin resistance. Skeletal muscles are responsible for $70-80 \%$ of whole body insulin-stimulated glucose uptake, and therefore the main organ affected by insulin resistance. Skeletal muscle also plays an important role in lipid metabolism. Plasma free fatty acid (FFA) concentration is often elevated in obese and type 2 diabetic subjects as compared to lean, healthy ones [1]. Elevated fatty acid (FA) uptake has been observed in skeletal muscle of individuals with T2D [2] obese humans [3] and subjects fed HFD [4]. After entering the cell, FA are activated to form a fatty acyl-CoA (LCACoA) and this step is catalyzed by the enzyme acyl-CoA synthetase (ACS). Then, the acyl-CoA are directed to the mitochondria for $\beta$-oxidation or synthesis of intramyocellular lipids (IMCL) [5]. LCACoA are transported through the mitochondrial membrane as acyl-carnitines that are synthesized by the enzyme carnitine palmitoyltransferase 1 (CPT1). Decreased content of CPT1 was observed in the skeletal muscle of obese individuals suggesting that the ability to direct FA towards $\beta$-oxidation is impaired in obesity. It is well known, that malonyl-CoA (a key intermediate in synthesis of fatty acids) inhibits CPT1 and in this way also mitochondrial uptake of FA. The elevated FA uptake by cell with decreased mitochondrial channeling of FA contributes to the accumulation of IMCL. The main groups of lipid which are currently considered to be responsible for induction of muscle IR are: LCACoA, DAG and Cer. Each of them is reported to inhibit the insulin signaling cascade. The accumulation of LCACoAs has been reported in obese, insulin resistant individuals [6] and during HFD feeding [7]. The increased content of intracellular DAG has been demonstrated in the insulin-resistant muscle of HFD rats [8] and Zucker rats [9]. Similarly, muscle Cer accumulation has been observed in muscles of obese, insulin-resistant humans [10] and obese insulin-resistant rats $[9,11]$. It has been found that LCACoAs and DAGs affect insulin signaling pathway through the activation of serine/threonine PKC isoenzymes [12], whereas Cer inhibits the pathway at Akt/PKB level [13]. Other sphingolipid species such as gangliosides [14] or sphingosine1-phosphate [15] may also contribute to IR.

The aim of this study was to elucidate the role of particular bioactive lipid species in fat-induce IR. We utilized stable isotope labeled $\left[\mathrm{U}-{ }^{13} \mathrm{C}\right]$ - palmitate tracer and ultra-highperformance liquid chromatography (UHPLC) - tandem mass spectrometer (LC/MS/MS) to follow the fate of FAs within the cell. Moreover, by the use of chemical inhibitor of Cer denovo synthesis (myriocin) together with an ability to measure FSR of particular lipids, we were able to establish the role of particular lipid class in triggering lipid-induced skeletal muscle IR. Present work answered several important questions concerning intracellular fate of plasma-borne FA, such as dynamics of cellular uptake, activation towards LCACoAs and incorporation into particular class of (IMCL) as well as its role in inhibition of insulin signaling pathway.

\section{Materials and Methods}

\section{Animals and study design}

The investigation was approved by the Institutional Animal Care and Use Committee of Medical University of Bialystok. The experiments were carried out on male Wistar rats, randomly divided into following groups: 1. Control, fed 8 weeks a control diet (Research Diets INC D12450B, 10\% calories from fat) $(\mathrm{n}=8)$. 2. HFD group, fed for 8 weeks high fat-diet (HFD) (Research Diets INC D12492, 60\% calories from saturated fat) $(n=8)$. 3. Myriocin-treated (HFD/Myr) $(n=6)$ fed for 8 weeks HFD with daily intraperitoneal injection of SPT inhibitor myriocin ( $0.5 \mathrm{mg} / \mathrm{kg}$, in PBS buffer). One day before infusion experiment, an oral glucose tolerance test (OGTT) and intraperitoneal insulin tolerance tests (ITT) were undertaken. On the last day of the study the $\left[\mathrm{U}-{ }^{13} \mathrm{C}\right]$-palmitate was infused for 2 hours into a tail vain. During the last 30 minutes, insulin $(0.5 \mathrm{U} / \mathrm{kg})$ was administrated through the catheter to measure the insulin-stimulated protein phosphorylation. The rats were anaesthetized by intraperitoneal injection of pentobarbital in a dose of 80 $\mathrm{mg} / \mathrm{kg}$ of body weight. The soleus was taken and frozen in liquid nitrogen and then stored at $-80^{\circ} \mathrm{C}$ until analysis.

\section{KARGER}




\section{Cellular Physiology Cell Physiol Biochem 2016;40:1207-1220 \begin{tabular}{l|l|l|} 
and BOI: 10.1159/000453174 & $\begin{array}{l}\text { C) 2016 The Author(s). Published by S. Karger AG, Basel } \\
\text { www.karger.com/cpb }\end{array}$
\end{tabular} \\ Blachnio-Zabielska et al.: Fat-Induced Muscle Insulin Resistance}

Table 1. Content of skeletal muscle ceramide molecular species in HFD-induced insulin resistance and under myriocin (HFD/Myr) treatment. Values are mean +/- SEM; p < 0.05 (pmol/mg); a -vs Control; *-vs HFD; $\mathrm{n}=8$ for Control and HFD, $\mathrm{n}=6$ for HFD $/ \mathrm{Myr}$

\begin{tabular}{lccccccccc}
\hline & C14:0-Cer & C16:0-Cer & C18:1-Cer & C18:0-Cer & C20:0-Cer & C22:0-Cer & C24:1-Cer & C24:0-Cer & $\begin{array}{c}\text { Total } \\
\text { Cer }\end{array}$ \\
\hline Control & $0.123 \pm 0.010$ & $4.41 \pm 0.18$ & $0.91 \pm 0.04$ & $6.74 \pm 0.45$ & $0.71 \pm 0.02$ & $0.71 \pm 0.02$ & $0.81 \pm 0.04$ & $3.75 \pm 0.13$ & $18.17 \pm 1.12$ \\
HFD & $0.047 \pm 0.001^{\mathrm{a}}$ & $3.41 \pm 0.11$ & $2.04 \pm 0.06^{\mathrm{a}}$ & $14.17 \pm 0.42^{\mathrm{a}}$ & $0.69 \pm 0.03$ & $0.68 \pm 0.02$ & $0.41 \pm 0.02^{\mathrm{a}}$ & $2.98 \pm 0.11 \mathrm{a}$ & $24.42 \pm 1.78 \mathrm{a}$ \\
HFD/Myr & $0.069 \pm 0.002^{\mathrm{a}^{*}}$ & $1.76 \pm 0.02^{\mathrm{a}^{*}}$ & $1.07 \pm 0.02^{*}$ & $7.04 \pm 0.13^{*}$ & $0.52 \pm 0.03^{\mathrm{a}^{*}}$ & $0.60 \pm 0.03^{\mathrm{a}}$ & $0.50 \pm 0.02^{\mathrm{a}}$ & $2.80 \pm 0.07 \mathrm{a}$ & $14.37 \pm 0.48^{\mathrm{a}^{*}}$ \\
\hline
\end{tabular}

Lipid concentration and enrichment measurements

Plasma FFA concentration and isotopic enrichment was measured by LC/MS according to Persson et al. [16]. Measured fatty acid species are listed in Table S2 (for all online suppl. material, see www.karger. com/doi/10.1159/000453174). Malonyl-CoA, LCACoA content and ${ }^{13} \mathrm{C}_{16}$-CoA enrichment was measured according to Blachnio-Zabielska et al. [17] with the use of LC/MS/MS. Quantified LCACoA species are listed in Table S3. The ceramide content and isotopic enrichment was measured using a UHPLC/MS/MS approach according to Blachnio-Zabielska et al. [18]. The content and isotopic enrichment of DAG was measured using a UHPLC/MS/MS approach according to Blachnio-Zabielska et al. [19]. Measured Cer and diacylglycerols molecular species are listed in Table 1 and Table S5, respectively. Acyl-carnitine concentration and isotopic enrichment $\left({ }^{13} \mathrm{C}_{16}\right.$-carnitine) was measured according to Sun et al. [20] with minor modification. Both concentration and isotopic enrichment were measured with the use of UHPLC/MS/MS. Table S4 provides measured acyl-carnitine species.

Isotope infusion, tissue collection and lipid fractional synthesis rate calculation (FSR)

The mixture of albumin-bound ${ }^{13} \mathrm{C}_{16}$-potassium-palmitate (uniformly labeled, Sigma-Aldrich, St. Louis, MO USA) was prepared as previously described [21]. Plasma palmitate concentration and isotopic enrichment in both the infusate and plasma samples was measured according to Persson et al. [16]. Isotope tracer infusion and lipid FSR calculation was performed according to Blachnio-Zabielska et al. [19]. The area under palmitate enrichment curve was identical in all experimental groups (see online suppl. material, Fig. S1).

Protein and RNA isolation

Total RNA and protein was isolated from muscle samples using NucleoSpin ${ }^{\circledR} \mathrm{RNA} /$ Protein isolation kit (Macherey-Nagel, Bethlehem, PA), according to procedure by Bahn et al. [22]. RNA and proteins were separated on NucleoSpin columns according to manufacturer guidelines.

Western Blot

Following target proteins were quantified using primary antibodies: GLUT4, CD36, CPT1A, (Abcam, Cambridge, MA), ACC, acyl-coenzyme A synthetase (ACS), SPT, Akt, pAktSer473, pAktThr308, AS160, AS160Thr642 and GAPDH (Santa Cruz Biotechnology). Values were normalized to GAPDH protein expression measured from parallel runs and expressed as fold changes over control group values. All chemicals and equipment used for immunoblotting were purchased from Bio-Rad (Hercules, CA).

RT-PCR

The RNA was reverse transcribed into cDNA using iScript cDNA Synthesis Kit (Bio-Rad, Hercules, CA) with oligo $(\mathrm{dT})_{18}$. Specific primers for the ACC, ACS, CD36, CPT1, FABPpm, FATP1, GLUT4, SPT and GAPDH were designed using the Beacon Designer Software. The housekeeping gene GAPDH was used as the reference gene for quantification. Quantitative real-time PCR was performed with SYBR Green Supermix Kit (Bio-Rad) using a Bio-Rad Chromo4 system.

Acyl-coenzyme A synthetase (ACSs) activity

Acyl-coenzyme A synthetase (ACSs) activity was assayed in muscle microsomes according to method by De Jong et al. [23], with the use of radiolabeled [9,10-3H(N)]-palmitate (American Radiolabeled Chemicals, Inc. Saint Louis, MO). 
Oral Glucose Tolerance Test (OGTT), Insulin Tolerance Test (ITT), plasma insulin and HOMA-IR

OGTT was performed in by oral glucose administration at a dose of $3 \mathrm{~g} / \mathrm{kg}$. ITT was performed by intraperitoneal injection of insulin in a dose of $0.75 \mathrm{U} / \mathrm{kg}$ body weight. Blood samples from tail vein were measured at given intervals by glucometer (AccuCheck, Roche. Germany). Plasma insulin was measured with an ELISA insulin assay (Rat/Mouse Insulin Millipore). HOMA-IR index value was calculated according to Cacho et al. [24].

Principal component analysis, correlation analysis and statistical significance estimation

PCA was performed using Statistica 10.0 software package as described earlier [25]. To prevent an artificial increase in the PCA model strength, we excluded a majority of closely interdependent variables. We used Pearson's approach with Bonferroni correction for multiple comparisons to establish relationships between selected variables chosen on the basis of PCA analysis. Statistical significance between groups was estimated using ANOVA with the Tukey honestly significant difference post-hoc test for unequal n-numbers. Significance level was set to $\mathrm{p}<0.05$.

\section{Results}

Whole-body insulin sensitivity

Animals fed HFD developed IR, which was manifested by elevated fasting blood glucose concentration, increased HOMA-IR index, reduced insulin responsiveness and impaired glucose tolerance (see online suppl. material, Table S1, Fig. 1). Myriocin normalized insulinrelated parameters to control values.

Plasma FFA concentration, muscle expression of FA transporters, ACS activity, LCACoA content and FSR

Total plasma FFA concentration increased by $30 \%$ in HFD group as compared to Control ( $p<0.05$ vs Control; Fig. 2A). Myriocin increased plasma FFA by $45 \%$ ( $p<0.05$ vs Control).

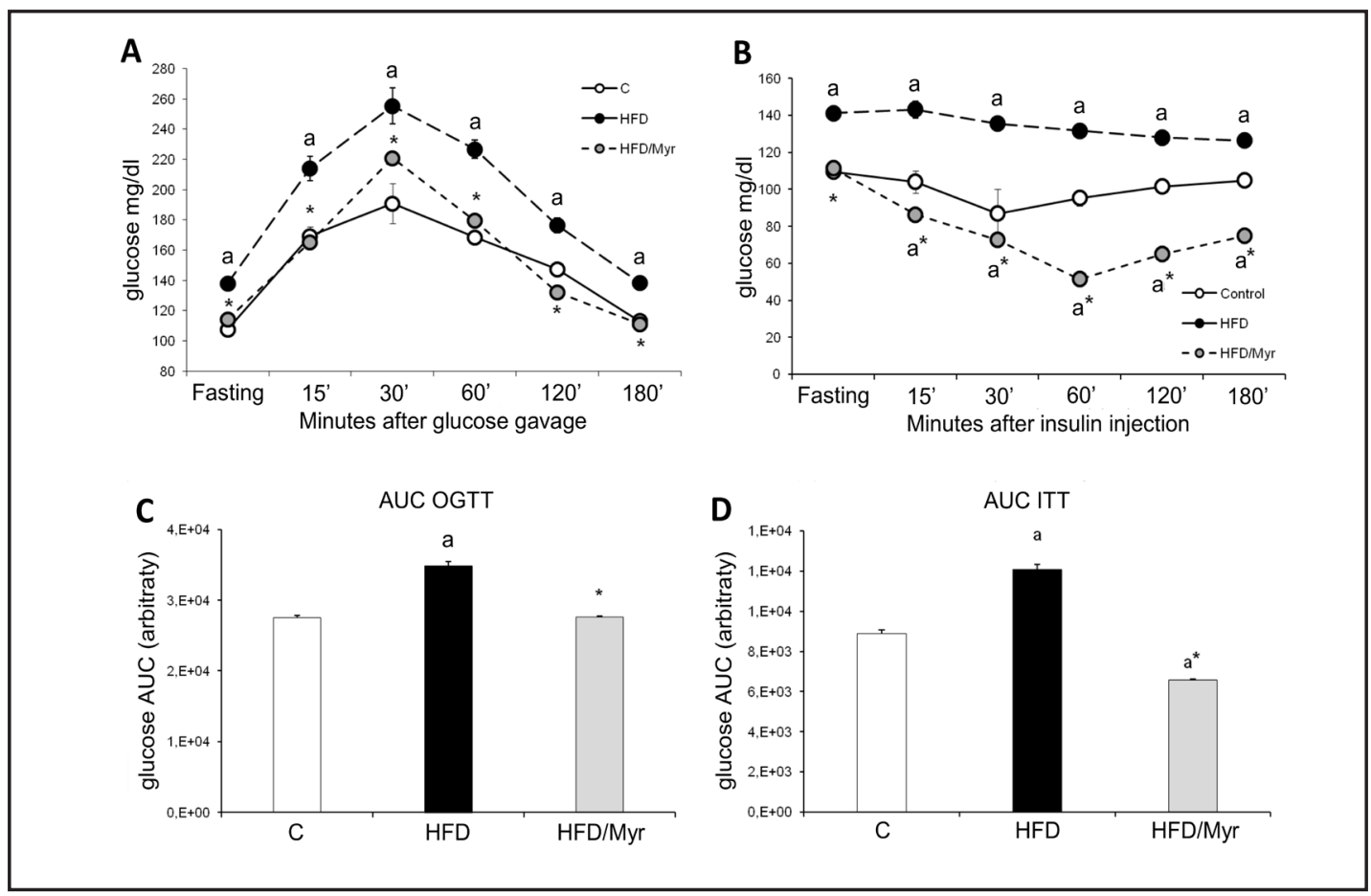

Fig. 1. Treatment with myriocin improves glucose and insulin tolerance in animals on high-fat diet. Panels A and B - OGTT and ITT tests respectively. Panels C and D - AUC values for OGTT and ITT tests, respectively. Values are mean +/- SD. $\mathrm{p}<0.05$ against: a- vs. C; *- vs. HFD. 
Fig. 2. The effect of metformin and myriocin on intracellular uptake and LCACoA conversion of fatty acids in skeletal muscle of HFD-fed animals. Panel A - plasma FFA; $B$ and $C$ - mRNA and protein expression of CD36, FATP1 and FABPpm. D - muscle LCACoA content; $\mathrm{E}$ and $\mathrm{F}$ - protein expression and enzymatic activity of ACS. Values are mean +/- SD. p $<0.05$ against: a- vs. C; ${ }^{*}$ - vs. HFD.

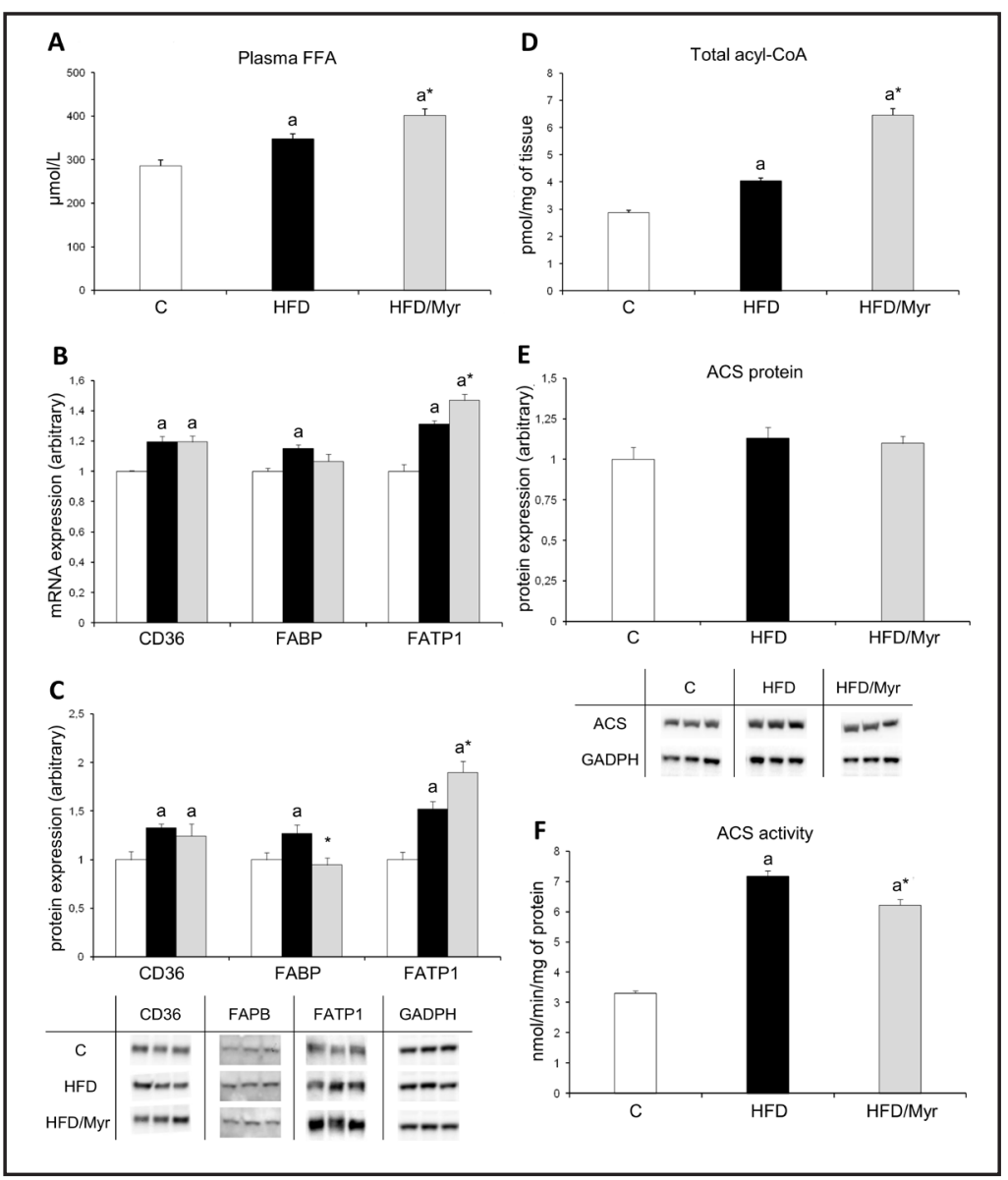

Both, HFD and HFD/Myr animals displayed significant increase in both, mRNA and protein level of CD36 and FATP1 ( $p<0.05$ vs Control, Fig. 2B and 3C). Accumulation of intramuscular LCACoA's was noted in both the HFD and HFD/Myr groups ( $p<0.05$ vs Control, Fig. 2D), yet myriocin treatment almost doubled the total LCACoA's in muscle, as compared to both the Control and HFD animals ( $\mathrm{p}<0.05)$. All the above changes in the content of LCACoA were most visible for C18:0-acyl-CoA (see online suppl. material, Table S3). Enzymatic activity of acyl-CoA synthase (ACS) increased significantly in both the HFD and HFD/Myr groups, yet there were no significant changes in (ACS) mRNA and protein content (Fig. S3 and Fig. 2E) or acyl-CoA FSR between experimental groups (see online suppl. material, Fig. S2).

\section{Mitochondrial channeling of fatty acids}

Both the HFD and HFD/Myr animals displayed significant decrease in FSR and content of acyl-carnitine ( $p<0.05$ vs Control, Fig. 3 A-D; Table S4, see online suppl. material). Moreover, in HFD group the expression of CPT1 was lower than respective values in the Control group ( $p<0.05$ vs Control). The same was noted for acyl-carnitine to LCA-CoA concentration ratio and respective FSR ratio (Fig. 3H, Fig. S4, see online suppl. material).

Muscle malonyl-CoA content doubled in HFD group as compared to Control group ( $p$ $<0.05$ vs Control; Fig. 3E). HFD decreased acetyl-CoA carboxylase (ACC) phosphorylation, which indicates its activation ( $p<0.05$ vs Control, Fig. 3G). Myriocin treatment restored the malonyl-CoA content to control values, yet had no effect on ACC gene expression or phosphorylation state, as compared with HFD animals.

\section{Muscle bioactive lipid profile}

HFD increased the content of muscle ceramide as compared to Control $(\mathrm{p}<0.05$, Fig. $4 \mathrm{~A}$ ), which was attributed entirely to stearoyl- and oleoyl- ceramide species (Table 1 ). The 
A

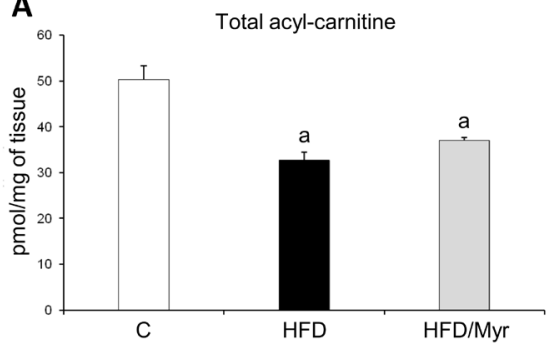

B Acyl-carnitine FSR

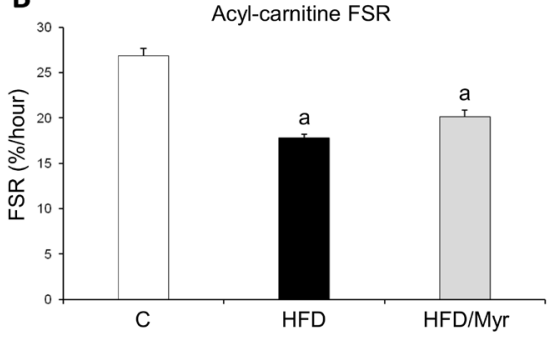

C

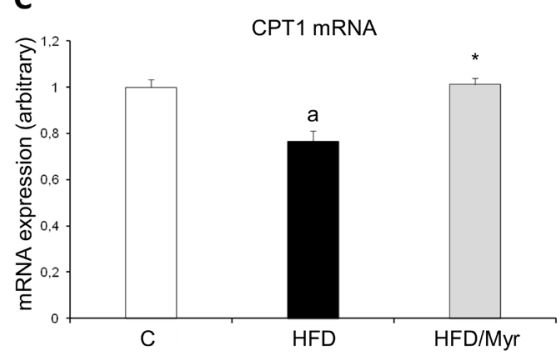

D

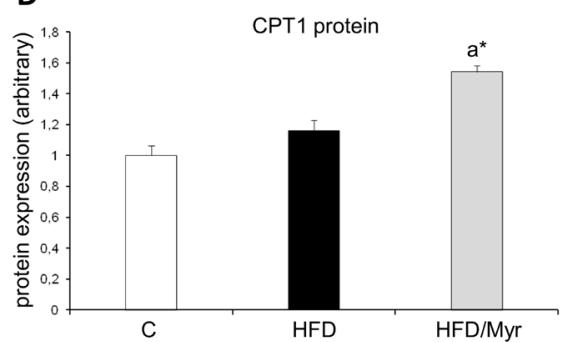

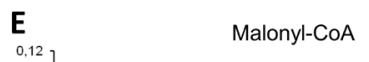

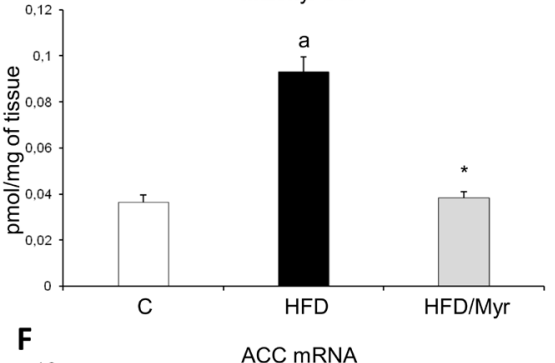

F ACC MRNA

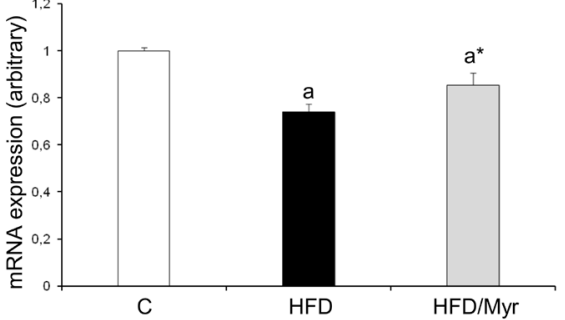

GACC/ACC
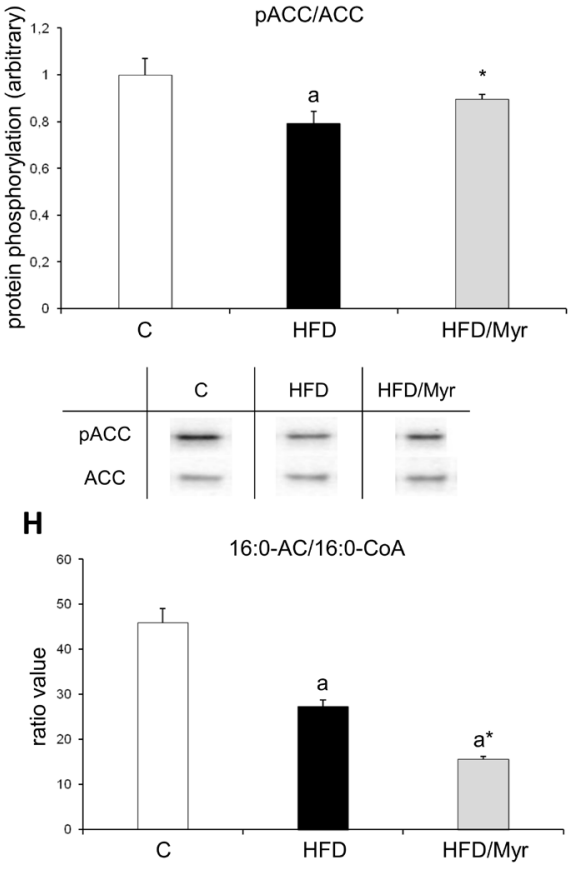

Fig. 3. High-fat diet alone or combined with myriocin suppresses acyl-carnitine FSR and decreases mitochondrial channeling of fatty acids. Panel A and B -total acyl-carnitine content and FSR; Panel C and D - mRNA and protein expression of CPT1. Panel E - malonyl-CoA content; Panels F and G - mRNA expression and protein phosphorylation of ACC. Panel H - 16:0-AC to 16:0-CoA ratio (a measure of mitochondrial fatty acids uptake). Values are mean $+/-$ SD. $p<0.05$ against: a- vs. C; ${ }^{*}$ - vs. HFD.

elevation was accompanied by up-regulation of SPT mRNA and protein level ( $p<0.05$ vs Control; Fig. 4C, D). Interestingly, Cer FSR was not affected (Fig. 4B). This disparity could be explained by the use of labeled palmitate $\left({ }^{13} \mathrm{C} 16: 0-\mathrm{FA}\right)$ for FSR estimation. The content of C16:0-Cer was not affected, whereas C18-derived ceramides were responsible for increased ceramide content in HFD group (Table 1). Myriocin did not normalized SPT expression, although significantly lowered both the content and FSR of ceramide $(\mathrm{p}<0.05$ vs Control 
Fig. 4. Myriocin treatment decreases ceramide content yet simultaneously increases diacylglycerol content in skeletal muscle of HFD animals. Panel A and $\mathrm{B}$ - ceramide content and FSR; C and D - mRNA and protein expression of SPT; Panels E, F and G DAG content and FSR of 16:0/16:0 DAG and 16:0 / $18: 1$ DAG, respectively. Values are mean +/SD. $p<0.05$ against: a- vs. C; ${ }^{*}$ - vs. HFD.
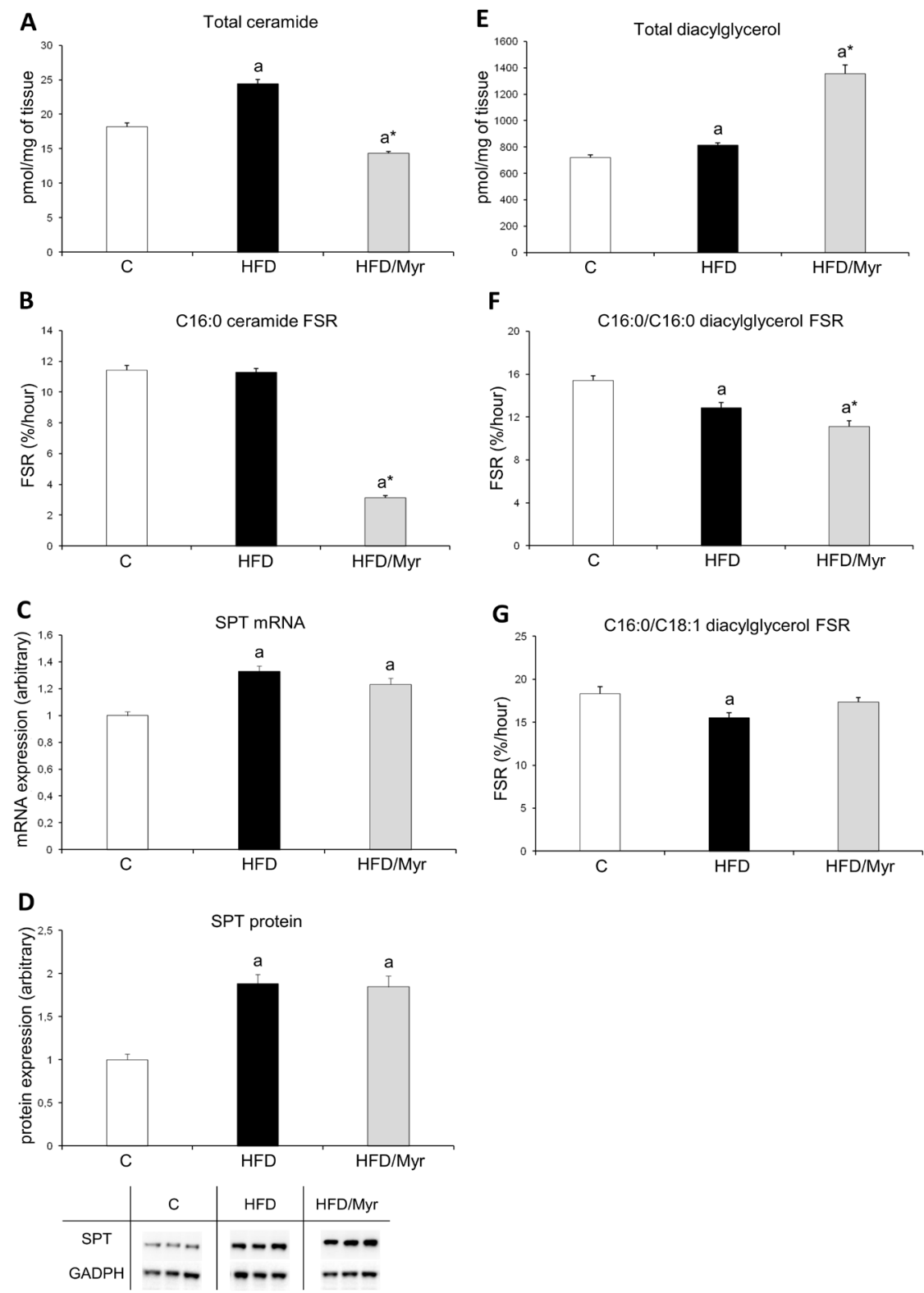

and HFD). In agreement with low ${ }^{13} \mathrm{C} 16: 0$-Cer FSR the content of C16:0-Cer also decreased in Myriocin treated animals. In HFD group total DAG content increased above Control values. Yet the content of 16/16-, 16/18:1-, 16/18-DAG decreased as compared to control group (see online suppl. material, Table S5), which is in agreement with decline in DAG FSR measured with ${ }^{13} \mathrm{C} 16: 0$-palmitate (Fig. 4F,G). In HFD/Myr animals, total DAG content doubled as compared to Control, with C18-derived DAGs responsible for majority of the change.

\section{Skeletal muscle insulin sensitivity}

In HFD animals, induction of IR was associated with decreased insulin action at the level of skeletal muscle, as suggested by decreased phosphorylation of Akt (Ser475 and Thr308), and AS160 under insulin stimulation (Fig. 5 A-C). Improvement of insulin-sensitivity in HFD/Myr was related to significant increase in insulin pathway proteins phosphorylation (Akt Ser475, Akt Thr308 and AS160), as compared to HFD animals ( $\mathrm{p}<0.05)$. There were no significant changes in mRNA of GLUT4 between studied groups (see online suppl. material, 
Fig. 5. Myriocin improves skeletal muscle insulin signaling in HFD-fed animals. Panel A and B - Akt/ PKB phosphotylation at serine 473 and theonine 308 , respectively; C and D - AS160 phosphorylation and GLUT4 expression, respectively. Values are mean +/- SD. p $<0.05$ against: a- vs. C; ${ }^{*}$ - vs. HFD.

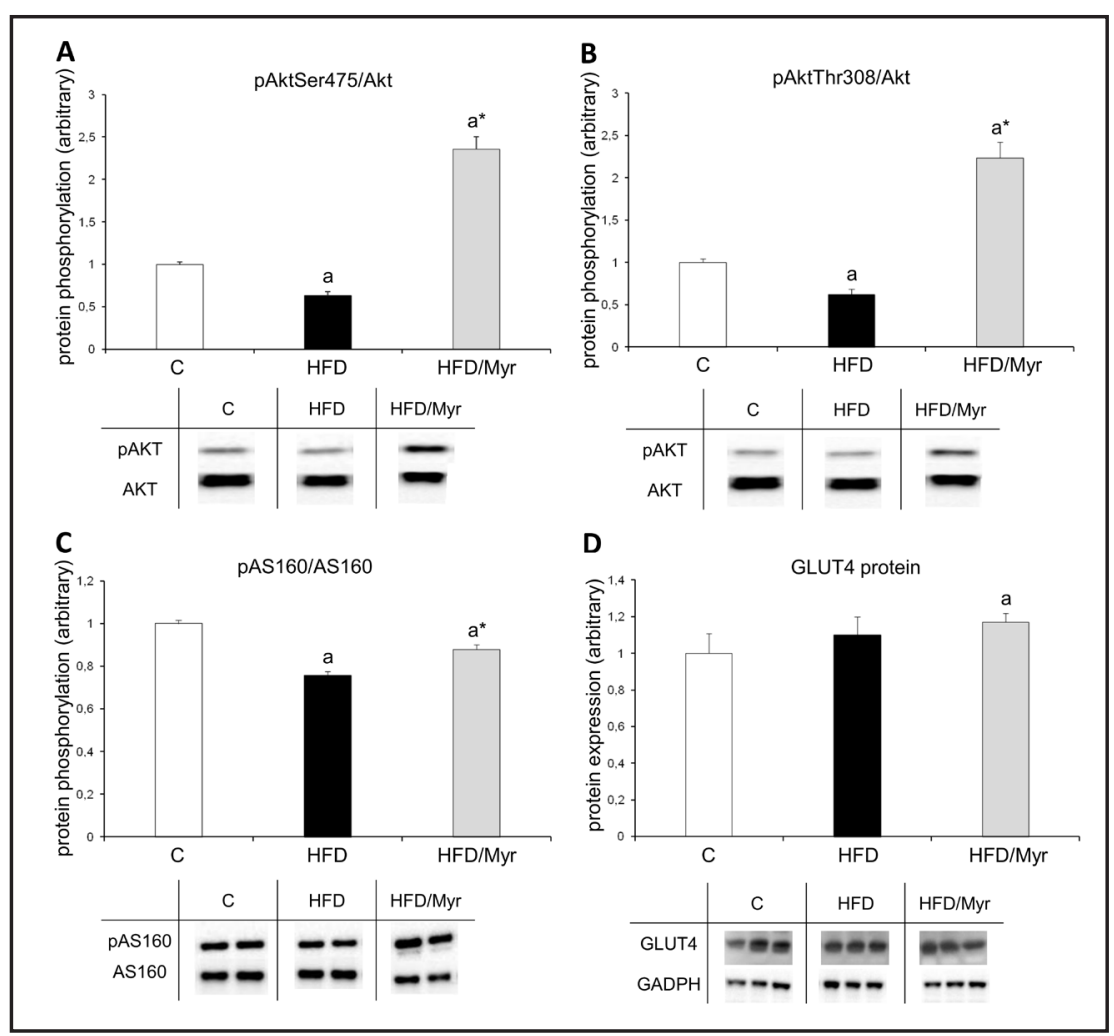

Fig. S5) and only slight but significant increased of protein content in HFD/Myr group (Fig. 5D).

Principal component (PCA) and correlation analysis

We employed PCA analysis to study relationships between individual lipid species and measures of the whole body and skeletal muscle insulin resistance. PCA analysis identified three major principal components, which were responsible for a total of $82.2 \%$ of cumulative variance within our data set $(\mathrm{pc} 1=43.3 \%, \mathrm{pc} 2=33.7 \%, \mathrm{pc} 3=5.2 \%$; cumulative $\mathrm{R} 2 \mathrm{X}=0.822$, Q2=0.674). Each experimental group clustered tightly on scores biplot (Fig. 6A). Overlay of variable vectors indicated that factors such as insulin-resistance measurements and stearoyl-, oleoyl- and total ceramide content were responsible for separation of HFD-only group from both the Control and HFD/Myr groups (along t2 axis, Fig. 6A). Total DAG, total LCA-CoA, their C18:1- derived molecular species and AKT phosphorylation state were responsible for separation of HFD/Myr animals from the other experimental groups (along t1 axis, Fig. 6A). Variables such as total content and FSR rate of acyl-carnitines and acyl-carnitine to LCA-CoA ratio were responsible for separation of Control group from both HFD-treated groups. Interestingly those variables included also short-chain (C14:0) and very long-chain (C24-based) ceramides. Loadings scatter plot revealed tight clustering of C18:0-, C18:1and total ceramide content with the measurements of insulin resistance such as HOMA-IR, fasting glucose, OGTT and ITT AUC (Feature cluster 1, Fig. 6B). Other cluster members were malonyl-CoA and C14:0-CoA. Lipid $\beta$-oxidation-related variables clustered together with acyl-carnitine content and FSR and short-chain and very-long chain ceramides and Control group representation (Feature cluster 2, Fig. 6B). Most of the individual DAG species and total DAG content clustered with total and C18:1-CoA level and AKT phosphorylation (Feature cluster 3, Fig. 6B). To further confirm interdependency between the muscular lipid species and IR parameters we used Pearson's r correlation estimation. Both, total and C18-chain length ceramides showed strong, positive correlation with HOMA-IR, OGTT AUC, ITT AUC and fasting plasma glucose ( $\mathrm{r}$ from 0.73 to $0.93, \mathrm{p}<0.001$ in all cases). Total ceramide displayed negative correlation with Akt phosphorylation state $(r=-0.83, p<0.001)$. Content and FSR 


\section{Cellular Physiology Cell Physiol Biochem 2016;40:1207-1220 \begin{tabular}{ll|l} 
DOI: 10.1159/000453174 & $\begin{array}{l}\text { O 2016 The Author(s). Published by S. Karger AG, Basel } \\
\text { wwww.karger.com/cpb }\end{array}$
\end{tabular} \\ Blachnio-Zabielska et al.: Fat-Induced Muscle Insulin Resistance}

Fig. 6. Principal component analysis (PCA) reveals association of insulin resistance measures with HFD-fed animals group and muscular 18:0-ceramide content, but not with myriocin-treated animals or muscular DAG accumulation. Panel A - scores scatter plot for $1^{\text {st }}$ and $2^{\text {nd }}$ PCA components. Arrows and names indicate direction of change and type and of major variables responsible for between-group differences. Panel B - loadings scatter plot for 2 first PCA components. The variables grouped in 3 major clusters (encircled) are described in results section.

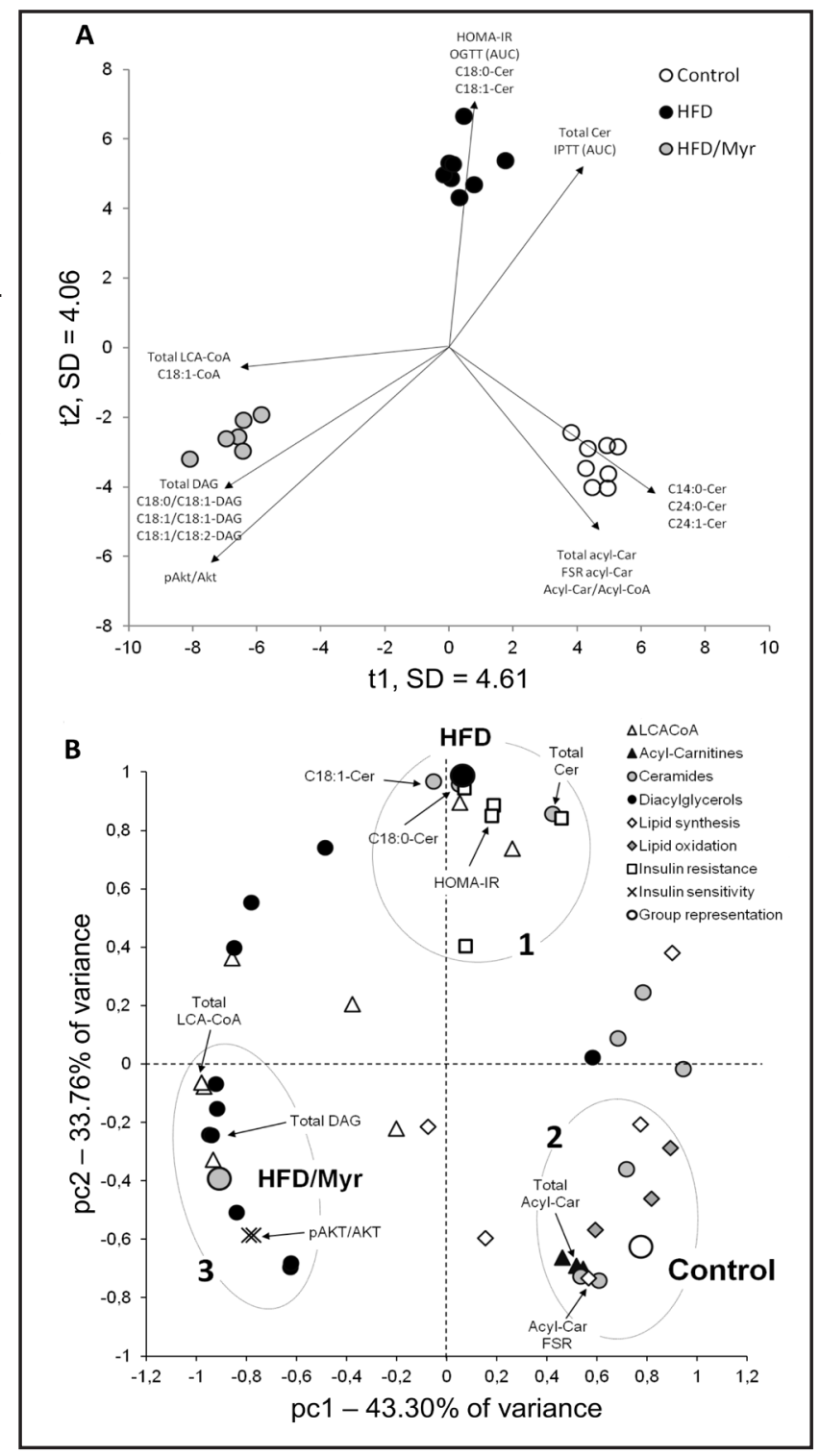

of palmitoyl-ceramide showed strong negative correlation only with Akt phosphorylation state ( $\mathrm{r}$ from -0.73 to $-0.94, \mathrm{p}<0.001$ ) but no other indicators of IR. Surprisingly, most of measured DAG species and total DAG content displayed positive correlation with muscular AKT phosphorylation ( $\mathrm{r}$ from 0.75 to $0.95, \mathrm{p}<0.001$ in all cases). Moreover C16:0/C18:1and 16:0/18:0-DAG negatively correlated with the OGTT AUC, ITT AUC and HOMA-IR ( $\mathrm{r}$ form -0.63 to $-0.71, \mathrm{p}<0.001$ in all cases), which suggest lack of DAG involvement in HFD-induced skeletal muscle insulin resistance. Acyl-carnitine FSR correlated negatively with OGTT ( $\mathrm{r}=$ $-0.70, \mathrm{p}<0.001$ ) whereas malonyl-CoA positively with OGTT AUC, ITT AUC, fasting plasma glucose and HOMA-IR values ( $\mathrm{r}$ from 0.74 to $0.96, \mathrm{p}<0.001$ in all cases).

\section{Discussion}

Our study was designed to establish which of the bioactive lipid species is the most important in HFD-induced muscle IR. Although the exact mechanism that leads to the development of IR in skeletal muscle is not fully understood, increased uptake of FA, intracellular fat accumulation and bioactive lipids have been shown to play a key role in 


\section{Cellular Physiology Cell Physiol Biochem 2016;40:1207-1220

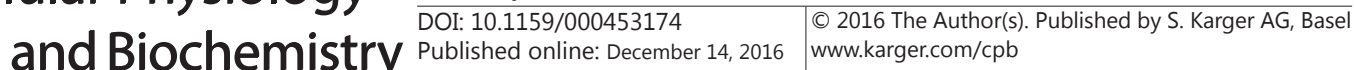 \\ Blachnio-Zabielska et al.: Fat-Induced Muscle Insulin Resistance}

skeletal muscle IR [26-28]. Currently, research focus shifts from total intramyocellular fat accumulation to bioactive lipid classes, and further to individual lipid species [29]. With the use of stable isotope tracers and mass spectrometry we followed the fate of plasma FA in HFD-induced intramuscular fat accumulation and IR. Our results indicate that C18:0 and C18:1-derived ceramides, as opposed to diacylglycerols, play a leading role in fat-induced skeletal muscle IR.

Increased rate of muscle FA uptake together with decreased mitochondrial channeling of FA may be responsible for IMCL accumulation. Elevated rates of FA uptake were observed in skeletal muscle of obese or T2DM animals [30-32]. We found that HFD feeding leads to accumulation of skeletal muscle acyl-CoA due to increased expression of FA transporters and ACS activity. Interestingly, myriocin treatment in HFD animals led to further increase in plasma FA and muscle acyl-CoA content. By blocking sphingoid production at systemic level, myriocin inhibits one of the metabolic pathways of plasma FFA disposal. This affects also intracellular acyl-CoA content which accumulates in skeletal muscle due to inhibition of sphingolipid biosynthesis. Analogous mechanisms were observed in plasma and skeletal muscle of CD36 [33] or CPT1b knock-out mice [34] where blocking of FA uptake to skeletal muscle or mitochondria led to plasma FA and intramuscular lipids accumulation. In the same way PGC-1 $\alpha$ protein ablation leads to intracellular TAG accumulation in L6 myotubes due to impaired mitochondrial FA metabolism [35]. Yet despite oversupply of the both plasma FA and muscle acyl-CoA myriocin-treated animals displayed improvement of IR at both systemic and muscular level which points to bioactive lipids as major contributors in IR.

Improvement of IR by augmentation of mitochondrial FA uptake is one of the molecular mechanisms of antidiabetic medication $[36,37]$. Thus reduction in muscle mitochondrial FA channeling could be responsible in part for induction of IR. Accumulation of intramuscular lipids due to CPT1 inhibition by excess of malonyl-CoA is often observed in IR state [3840]. Other studies indicate, that incomplete mitochondrial FA oxidation, manifested by high content of acyl-carnitines, contributes to skeletal muscle IR [41]. In our study HFD lead to decrease in acyl-carnitine content, acyl-carnitine FSR and acyl-carnitine to LCACoA ratio. Moreover, we observed activation of ACC and accumulation of malonyl-CoA. It has to be noted that accumulation of acyl-carnitines in not universal under lipid overload in both experimental animals [42] and humans [43], and this discrepancy could be explained by factors such as different experimental models, chow composition or species. Despite differences both mechanisms support thesis, that in IR muscle, mitochondrial FA processing is not sufficient for the high rate of cellular FA uptake, which leads to accumulation of intracellular LCACoAs and subsequently other lipids. Interestingly, improved insulin sensitivity in myriocin-treatmed animals was not associated with improved mitochondrial channeling of FA, as reflected in lower content and FSR of acyl-carnities and acyl-carnitine to LCACoA ratio. We did not observe detrimental effects of LCACoA accumulation on skeletal muscle insulin sensitivity, which points to other intramuscular lipid species as the inducers of IR.

Although strong link between the intracellular DAG accumulation and insulin resistance has been previously described in-vitro $[44,45]$ and in-vivo in obesity and insulin resistant states $[8,9,46]$, in a number of the studies relationship was not observed [47]. In our study increase in intramuscular DAG in HFD animals was moderate and strongly varied with acyl chain composition. Only C18:0, C18:1 and C18:2-derived molecular species displayed elevated levels, which is related to both the plasma FFA and muscle LCACoA species bioavailability. Similar DAG species composition was reported by Szendroedi et al. in obese or T2D subjects [48]. Yet the study by Selathurai et al. shows that even twofold increase in above DAG molecular species due to phosphatidylethanolamine synthesis inhibition (CTP:phosphoethanolamine cytidylyltransferase knock-out) does not affect skeletal muscle insulin sensitivity [47]. We achieved similar outcome in myriocin-treated HFD animals by re-routing LCACoA flux from de novo sphingolipids synthesis towards DAG synthesis. Nevertheless, despite significant accumulation of intramuscular LCACoA and DAG, myriocin-treated animals show improvement in insulin sensitivity and significant decrease 


\section{Cellular Physiology Cell Physiol Biochem 2016;40:1207-1220 \begin{tabular}{ll|l} 
DOI: 10.1159/000453174 & $\begin{array}{l}\text { O 2016 The Author(s). Published by S. Karger AG, Basel } \\
\text { www.karger.com/cpb }\end{array}$
\end{tabular} \\ Blachnio-Zabielska et al.: Fat-Induced Muscle Insulin Resistance}

in ceramide content. This observation strongly supports the involvement of ceramide, as opposed to DAG, in fat-induced skeletal muscle IR. In our study intramuscular ceramide accumulation in HFD animals was accompanied by IR. However, not all Cer species were equally affected. Only C18:0-Cer and C18:1-Cer species were elevated whereas C16:0-Cer remained stable. Recent studies link C16:0-Cer with hepatic insulin resistance $[49,50]$ whereas skeletal muscle C18-derived ceramides account for majority of Cer accumulation in HFD animals [51], mice with uncontrolled STZ-diabetes [52] and in obese, diabetic patients [53]. Inhibition of de novo sphingolipid synthesis by myriocin also had greatest impact on C18:0- and C18:1-Cer species and was accompanied by significant improvement in both the whole-body and skeletal muscle insulin sensitivity. Despite Myr treatment SPT expression was equal in both HFD and HFD/Myr animals. Myriocin acts as suicide inhibitor of SPT by covalent modification of essential catalytic serine, thus does not affect SPT expression but completely bocks its enzymatic activity at low nanomolar concentrations [54].

The next goal of our study was to elucidate the role of the accumulation of particular lipid species on insulin action and muscle insulin sensitivity. Previously published works demonstrated that all of the studied lipid species (LCACoA, DAG and Cer) negatively affect the insulin pathway [7, 12, 55-58]. In HFD animals intramuscular lipid accumulation was visible in all above lipid classes and coincided with both local (Akt and AS160 phosphorylation) and systemic (HOMA-IR, OGTT and ITT) measures of insulin resistance. Yet significant accumulation of LCACoA and DAG in skeletal muscle of HFD/Myr animals, without adverse effects on insulin sensitivity, points at ceramide as the key lipid in induction of muscular IR. Moreover, our data indicate that IR parameters show strong correlation with muscular C18:0-Cer and C18:1-Cer content whereas relationship between DAG and IR was not visible.

Taking together, HFD leads to induction of IR manifested by increased HOMA-IR and declined response to glucose or insulin. At skeletal muscle level HFD consumption decreased both the Akt and AS160 phosphorylation (Ser473 and Thr308) as compared to control. Moreover, HFD led to increased plasma FFA concentration, and inhibited mitochondrial uptake of FA through excessive production of malonyl-CoA. In consequence, we have observed an accumulation of LCACoA, DAGs, and ceramides. Myriocin improved insulin sensitivity by enhance Akt and AS160 phosphorylation. The insulin-sensitizing effects of myriocin were accompanied by decrease in C18:0- and C18:1 ceramide. Myriocin treatment led to the improvement in insulin sensitivity through inhibition of de-novo ceramide synthesis. Although myriocin caused elevated LCACoA and DAG level, it did not affect insulin sensitivity. Our results indicate that accumulation of stearoyl and oleoyl-ceramide species plays the major role in suppressing muscle insulin action.

\section{Abbreviations}

ACC (Acetyl-CoA carboxylase); ACS (Acyl-coenzyme A synthetase); Akt/PKB (Protein kinase B); AMPK (5' adenosine monophosphate-activated protein kinase); AS-160 (Akt substrate of $160 \mathrm{kDa}$ ); Cer (Ceramide); CD36 (Fatty acid translocase); CPT-1 (Carnitine palmitoyltransferase-1) DAG (Diacylglycerol); FA (Fatty acids); FABPpm (Plasma membrane fatty acid binding protein); FATP1 (Fatty acid transport protein 1); FSR (Fractional synthesis rate); GAPDH (Glyceraldehyde 3-phosphate dehydrogenase); GLUT4 (Glucose transporter type 4); HFD (High fat diet); IMCL (Intramyocellular lipids); LCACoA (Long-chain acylCoA); Myr (Myriocin); PKC (Protein kinase C); PPA2 (Protein phosphatase 2A); SPT (Serine palmitoyltransferase).

\section{Funding}

This work was supported by Foundation for Polish Science Grant HOMING PLUS/2010$2 / 1$, and by the Medical University of Bialystok (Grants 153-18698, 153-18699 and 15318697).

\section{KARGER}




\section{Cellular Physiology Cell Physiol Biochem 2016;40:1207-1220 \begin{tabular}{l|l|l} 
and Biochemistry $10.1159 / 000453174$ & $\begin{array}{l}\text { DO } 2016 \text { The Author(s). Published by S. Karger AG, Basel } \\
\text { Published online: December 14, } 2016\end{array}$ \\
www.karger.com/cpb
\end{tabular} \\ Blachnio-Zabielska et al.: Fat-Induced Muscle Insulin Resistance}

\section{Disclosure Statement}

The authors declare that they have no competing interests.

\section{References}

1 Belfort R, Mandarino L, Kashyap S, Wirfel K, Pratipanawatr T, Berria R, Defronzo RA, Cusi K: Dose-response effect of elevated plasma free fatty acid on insulin signaling. Diabetes 2005;54:1640-1648.

2 Bruce CR, Anderson MJ, Carey AL, Newman DG, Bonen A, Kriketos AD, Cooney GJ, Hawley JA: Muscle oxidative capacity is a better predictor of insulin sensitivity than lipid status. J Clin Endocrinol Metab 2003;88:5444-5451.

3 Simoneau JA, Veerkamp JH, Turcotte LP, Kelley DE: Markers of capacity to utilize fatty acids in human skeletal muscle: relation to insulin resistance and obesity and effects of weight loss. FASEB J 1999;13:20512060.

4 Roepstorff C, Helge JW, Vistisen B, Kiens B: Studies of plasma membrane fatty acid-binding protein and other lipid-binding proteins in human skeletal muscle. Proc Nutr Soc 2004;63:239-244.

5 Jeukendrup AE: Regulation of fat metabolism in skeletal muscle. Ann N Y Acad Sci 2002;967:217-235.

6 Hulver MW, Berggren JR, Cortright RN, Dudek RW, Thompson RP, Pories WJ, MacDonald KG, Cline GW, Shulman GI, Dohm GL, Houmard JA: Skeletal muscle lipid metabolism with obesity. Am J Physiol Endocrinol Metab 2003;284:E741-747.

7 Ellis BA, Poynten A, Lowy AJ, Furler SM, Chisholm DJ, Kraegen EW, Cooney GJ: Long-chain acyl-CoA esters as indicators of lipid metabolism and insulin sensitivity in rat and human muscle. Am J Physiol Endocrinol Metab 2000;279:E554-560.

8 Schmitz-Peiffer C, Browne CL, Oakes ND, Watkinson A, Chisholm DJ, Kraegen EW, Biden TJ: Alterations in the expression and cellular localization of protein kinase $C$ isozymes epsilon and theta are associated with insulin resistance in skeletal muscle of the high-fat-fed rat. Diabetes 1997;46:169-178.

9 Turinsky J, O'Sullivan DM, Bayly BP: 1,2-Diacylglycerol and ceramide levels in insulin-resistant tissues of the rat in vivo. J Biol Chem 1990;265:16880-16885.

10 Adams JM, Pratipanawatr T, Berria R, Wang E, DeFronzo RA, Sullards MC, Mandarino LJ: Ceramide content is increased in skeletal muscle from obese insulin-resistant humans. Diabetes 2004;53:25-31.

11 Harasim E, Stepek T, Konstantynowicz-Nowicka K, Baranowski M, Gorski J, Chabowski A: Myocardial lipid profiling during time course of high fat diet and its relationship to the expression of fatty acid transporters. Cell Physiol Biochem 2015;37:1147-1158.

12 Itani SI, Ruderman NB, Schmieder F, Boden G: Lipid-induced insulin resistance in human muscle is associated with changes in diacylglycerol, protein kinase C, and IkappaB-alpha. Diabetes 2002;51:20052011.

13 Schmitz-Peiffer C, Craig DL, Biden TJ: Ceramide generation is sufficient to account for the inhibition of the insulin-stimulated PKB pathway in C2C12 skeletal muscle cells pretreated with palmitate. J Biol Chem 1999;274:24202-24210.

14 Lipina C, Hundal HS: Ganglioside GM3 as a gatekeeper of obesity-associated insulin resistance: Evidence and mechanisms. FEBS Lett 2015;589:3221-3227.

15 Fayyaz S, Japtok L, Kleuser B: Divergent role of sphingosine 1-phosphate on insulin resistance. Cell Physiol Biochem 2014;34:134-147.

16 Persson XM, Blachnio-Zabielska AU, Jensen MD: Rapid measurement of plasma free fatty acid concentration and isotopic enrichment using LC/MS. J Lipid Res 2010;51:2761-2765.

17 Blachnio-Zabielska AU, Koutsari C, Jensen MD: Measuring long-chain acyl-coenzyme A concentrations and enrichment using liquid chromatography/tandem mass spectrometry with selected reaction monitoring. Rapid Commun Mass Spectrom 2011;25:2223-2230.

18 Blachnio-Zabielska AU, Persson XM, Koutsari C, Zabielski P, Jensen MD: A liquid chromatography/ tandem mass spectrometry method for measuring the in vivo incorporation of plasma free fatty acids into intramyocellular ceramides in humans. Rapid Commun Mass Spectrom 2012;26:1134-1140.

19 Blachnio-Zabielska AU, Zabielski P, Jensen MD: Intramyocellular diacylglycerol concentrations and [U-13C] palmitate isotopic enrichment measured by LC/MS/MS. J Lipid Res 2013;54:1705-1711. 


\section{Cellular Physiology Cell Physiol Biochem 2016;40:1207-1220 \begin{tabular}{l|l|l} 
and Biochemistry & $\begin{array}{l}\text { DOI: 10.1159/000453174 } \\
\text { Published online: December 14, } 2016\end{array}$ & $\begin{array}{l}\text { C) 2016 The Author(s). Published by S. Karger AG, Basel } \\
\text { www.karger.com/cpb }\end{array}$ \\
\hline
\end{tabular}}

20 Sun D, Cree MG, Zhang XJ, Bøersheim E, Wolfe RR: Measurement of stable isotopic enrichment and concentration of long-chain fatty acyl-carnitines in tissue by HPLC-MS. J Lipid Res 2006;47:431-439.

21 Guo Z, Nielsen S, Burguera B, Jensen MD: Free fatty acid turnover measured using ultralow doses of [U-13C]palmitate. J Lipid Res 1997;38:1888-1895.

22 Bahn A, Hagos Y, Reuter S, Balen D, Brzica H, Krick W, Burckhardt BC, Sabolic I, Burckhardt G: Identification of a new urate and high affinity nicotinate transporter, hOAT10 (SLC22A13). J Biol Chem 2008;283:1633216341.

23 De Jong H, Neal AC, Coleman RA, Lewin TM: Ontogeny of mRNA expression and activity of long-chain acylCoA synthetase (ACSL) isoforms in Mus musculus heart. Biochim Biophys Acta 2007;1771:75-82.

24 Cacho J, Sevillano J, de Castro J, Herrera E, Ramos MP: Validation of simple indexes to assess insulin sensitivity during pregnancy in Wistar and Sprague-Dawley rats. Am J Physiol Endocrinol Metab 2008;295:E1269-1276.

25 Zabielski P, Baranowski M, Błachnio-Zabielska A, Zendzian-Piotrowska M, Górski J: The effect of high-fat diet on the sphingolipid pathway of signal transduction in regenerating rat liver. Prostaglandins Other Lipid Mediat 2010;93:75-83.

26 Erion DM, Shulman GI: Diacylglycerol-mediated insulin resistance. Nat Med 2010;16:400-402.

27 Holland WL, Knotts TA, Chavez JA, Wang LP, Hoehn KL, Summers SA: Lipid mediators of insulin resistance. Nutr Rev 2007;65:S39-46.

28 Kraegen EW, Cooney GJ: Free fatty acids and skeletal muscle insulin resistance. Curr Opin Lipidol 2008;19:235-241.

29 Kitessa SM, Abeywardena MY: Lipid-Induced Insulin Resistance in Skeletal Muscle: The Chase for the Culprit Goes from Total Intramuscular Fat to Lipid Intermediates, and Finally to Species of Lipid Intermediates. Nutrients 2016;8

30 Chabowski A, Chatham JC, Tandon NN, Calles-Escandon J, Glatz JF, Luiken JJ, Bonen A: Fatty acid transport and FAT/CD36 are increased in red but not in white skeletal muscle of ZDF rats. Am J Physiol Endocrinol Metab 2006;291:E675-682.

31 Luiken JJ, Arumugam Y, Dyck DJ, Bell RC, Pelsers MM, Turcotte LP, Tandon NN, Glatz JF, Bonen A: Increased rates of fatty acid uptake and plasmalemmal fatty acid transporters in obese Zucker rats. J Biol Chem 2001;276:40567-40573.

32 Turcotte LP, Swenberger JR, Zavitz Tucker M, Yee AJ: Increased fatty acid uptake and altered fatty acid metabolism in insulin-resistant muscle of obese Zucker rats. Diabetes 2001;50:1389-1396.

33 Koonen DP, Sung MM, Kao CK, Dolinsky VW, Koves TR, Ilkayeva O, Jacobs RL, Vance DE, Light PE, Muoio DM, Febbraio M, Dyck JR: Alterations in skeletal muscle fatty acid handling predisposes middle-aged mice to diet-induced insulin resistance. Diabetes 2010;59:1366-1375.

34 Wicks SE, Vandanmagsar B, Haynie KR, Fuller SE, Warfel JD, Stephens JM, Wang M, Han X, Zhang J, Noland RC, Mynatt RL: Impaired mitochondrial fat oxidation induces adaptive remodeling of muscle metabolism. Proc Natl Acad Sci U S A 2015;112:E3300-3309.

35 Lukaszuk B, Miklosz A, Chabowski A, Gorski J: Modest decrease in PGC1alpha results in TAG accumulation but not in insulin resistance in L6 myotubes. Cell Physiol Biochem 2015;35:1609-1622.

36 Bhattacharjee S, Das N, Mandala A, Mukhopadhyay S, Roy SS: Fenofibrate Reverses Palmitate Induced Impairment in Glucose Uptake in Skeletal Muscle Cells by Preventing Cytosolic Ceramide Accumulation. Cell Physiol Biochem 2015;37:1315-1328.

37 Baranowski M, Zabielski P, Blachnio-Zabielska AU, Harasim E, Chabowski A, Gorski J: Insulin-sensitizing effect of LXR agonist T0901317 in high-fat fed rats is associated with restored muscle GLUT4 expression and insulin-stimulated AS160 phosphorylation. Cell Physiol Biochem 2014;33:1047-1057.

38 Dobbins RL, Szczepaniak LS, Bentley B, Esser V, Myhill J, McGarry JD: Prolonged inhibition of muscle carnitine palmitoyltransferase-1 promotes intramyocellular lipid accumulation and insulin resistance in rats. Diabetes 2001;50:123-130.

39 Sidossis LS, Mittendorfer B, Chinkes D, Walser E, Wolfe RR: Effect of hyperglycemia-hyperinsulinemia on whole body and regional fatty acid metabolism. Am J Physiol 1999;276:E427-434.

40 Rasmussen BB, Holmbäck UC, Volpi E, Morio-Liondore B, Paddon-Jones D, Wolfe RR: Malonyl coenzyme A and the regulation of functional carnitine palmitoyltransferase- 1 activity and fat oxidation in human skeletal muscle. J Clin Invest 2002;110:1687-1693. 


\section{Cellular Physiology Cell Physiol Biochem 2016;40:1207-1220

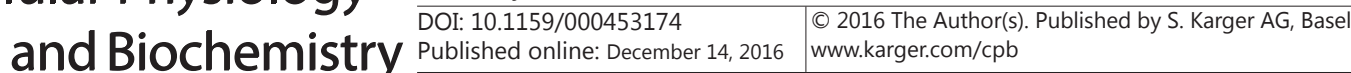

Blachnio-Zabielska et al.: Fat-Induced Muscle Insulin Resistance

41 Koves TR, Ussher JR, Noland RC, Slentz D, Mosedale M, Ilkayeva O, Bain J, Stevens R, Dyck JR, Newgard CB, Lopaschuk GD, Muoio DM: Mitochondrial overload and incomplete fatty acid oxidation contribute to skeletal muscle insulin resistance. Cell Metab 2008;7:45-56.

42 Alam N, Saggerson ED: Malonyl-CoA and the regulation of fatty acid oxidation in soleus muscle. Biochem J 1998;334:233-241.

43 Soeters MR, Sauerwein HP, Duran M, Wanders RJ, Ackermans MT, Fliers E, Houten SM, Serlie MJ: Muscle acylcarnitines during short-term fasting in lean healthy men. Clin Sci (Lond) 2009;116:585-592.

44 Lee JS, Pinnamaneni SK, Eo SJ, Cho IH, Pyo JH, Kim CK, Sinclair AJ, Febbraio MA, Watt MJ: Saturated, but not n-6 polyunsaturated, fatty acids induce insulin resistance: role of intramuscular accumulation of lipid metabolites. J Appl Physiol 2006;100:1467-1474.

45 Newsom SA, Everett AC, Park S, Van Pelt DW, Hinko A, Horowitz JF: Lipid mixtures containing a very high proportion of saturated fatty acids only modestly impair insulin signaling in cultured muscle cells. PLoS One 2015; 10:e0120871.

46 Oakes ND, Kennedy CJ, Jenkins AB, Laybutt DR, Chisholm DJ, Kraegen EW: A new antidiabetic agent, BRL 49653, reduces lipid availability and improves insulin action and glucoregulation in the rat. Diabetes 1994;43:1203-1210.

47 Selathurai A, Kowalski GM, Burch ML, Sepulveda P, Risis S, Lee-Young RS, Lamon S, Meikle PJ, Genders AJ, McGee SL, Watt MJ, Russell AP, Frank M, Jackowski S, Febbraio MA, Bruce CR: The CDP-Ethanolamine Pathway Regulates Skeletal Muscle Diacylglycerol Content and Mitochondrial Biogenesis without Altering Insulin Sensitivity. Cell Metab 2015;21:718-730.

48 Szendroedi J, Yoshimura T, Phielix E, Koliaki C, Marcucci M, Zhang D, Jelenik T, Muller J, Herder C, Nowotny P, Shulman GI, Roden M: Role of diacylglycerol activation of PKCtheta in lipid-induced muscle insulin resistance in humans. Proc Natl Acad Sci U S A 2014;111:9597-9602.

49 Turpin SM, Nicholls HT, Willmes DM, Mourier A, Brodesser S, Wunderlich CM, Mauer J, Xu E, Hammerschmidt P, Bronneke HS, Trifunovic A, LoSasso G, Wunderlich FT, Kornfeld JW, Bluher M, Kronke M, Bruning JC: Obesity-induced CerS6-dependent C16:0 ceramide production promotes weight gain and glucose intolerance. Cell Metab 2014;20:678-686.

50 Raichur S, Wang ST, Chan PW, Li Y, Ching J, Chaurasia B, Dogra S, Ohman MK, Takeda K, Sugii S, PewznerJung Y, Futerman AH, Summers SA: CerS2 haploinsufficiency inhibits beta-oxidation and confers susceptibility to diet-induced steatohepatitis and insulin resistance. Cell Metab 2014;20:687-695.

51 Lanza IR, Blachnio-Zabielska A, Johnson ML, Coenen-Schimke JM, Jakaitis DR, Lebrasseur NK, Jensen MD, Nair KS, Zabielski P: Influence of Fish Oil on Skeletal Muscle Mitochondrial Energetics and Lipid Metabolites during High-Fat Diet. Am J Physiol Endocrinol Metab 2013

52 Zabielski P, Blachnio-Zabielska A, Lanza IR, Gopala S, Manjunatha S, Jakaitis DR, Persson XM, Gransee J, Klaus KA, Schimke JM, Jensen MD, Nair KS: Impact of insulin deprivation and treatment on sphingolipid distribution in different muscle subcellular compartments of streptozotocin-diabetic C57Bl/6 mice. Am J Physiol Endocrinol Metab 2014;306:E529-542.

53 Bergman BC, Brozinick JT, Strauss A, Bacon S, Kerege A, Bui HH, Sanders P, Siddall P, Wei T, Thomas MK, Kuo MS, Perreault L: Muscle sphingolipids during rest and exercise: a C18:0 signature for insulin resistance in humans. Diabetologia 2016

54 Wadsworth JM, Clarke DJ, McMahon SA, Lowther JP, Beattie AE, Langridge-Smith PR, Broughton HB, Dunn TM, Naismith JH, Campopiano DJ: The chemical basis of serine palmitoyltransferase inhibition by myriocin. J Am Chem Soc 2013;135:14276-14285.

55 Cooney GJ, Thompson AL, Furler SM, Ye J, Kraegen EW: Muscle long-chain acyl CoA esters and insulin resistance. Ann N Y Acad Sci 2002;967:196-207.

56 Powell DJ, Turban S, Gray A, Hajduch E, Hundal HS: Intracellular ceramide synthesis and protein kinase Czeta activation play an essential role in palmitate-induced insulin resistance in rat L6 skeletal muscle cells. Biochem J 2004;382:619-629.

57 Straczkowski M, Kowalska I, Baranowski M, Nikolajuk A, Otziomek E, Zabielski P, Adamska A, Blachnio A, Gorski J, Gorska M: Increased skeletal muscle ceramide level in men at risk of developing type 2 diabetes. Diabetologia 2007;50:2366-2373.

58 Chavez JA, Summers SA: Characterizing the effects of saturated fatty acids on insulin signaling and ceramide and diacylglycerol accumulation in 3T3-L1 adipocytes and C2C12 myotubes. Arch Biochem Biophys 2003;419:101-109. 